\title{
FIGO Stage III Gestational Trophoblastic
} Tumor

National Cancer Institute

\section{Source}

National Cancer Institute. FIGO Stage III Gestational Trophoblastic Tumor. NCI

Thesaurus. Code C6400.

Lesion affects the lungs, with or without known genital structures invasion. 